A full account of all we know of the South African ape-men has been prepared by Dr. G. W. H. Schepers and myself, and it is hoped that this work will be published this year.

Transvaal Museum,
Pretoria.

R. Ввоом.

Jan. 27.

\section{Index of Diversity as Applied to Ecological Problems}

Is Research Items in Nature of December 30, p. 833, attention is directed to Dr. C. B. Williams' recent paper ${ }^{1}$ in which he applies the 'index of diversity' $(\alpha)$ to populations of various kinds. $\mathrm{He}$ claims that $\alpha$ is a property of the population, and is independent of sample size. He attempts to use it for the relation between numbers of species and size of sample in samples of plant populations obtained by means of quadrats, and in using some figures of Gleason's implies that, given a random distribution of quadrats, the application of $\alpha$ would be valid.

It is difficult to test these assumptions directly, since it is usually impossible to count individuals of plants, and therefore impossible to obtain the relation between numbers of species and numbers of individuals. Recently, while engaged on work for the Forestry Commission, I obtained figures for the composition of various kinds of heath vegetation, using quadrats of different sizes. The figures obtained show that the index of diversity varied both with the way in which the quadrats were distributed (random or otherwise) and with the quadrat size. Thus $\alpha$ obtained by using quadrats of $1 / 8$ square metre (quadrats being grouped together to give the varying sample sizes) was about 7 , while $\alpha$ derived from counts of the mean numbers of species in quadrats of three different sizes ( $1 / 20,1 / 8$ and 1 square metre)

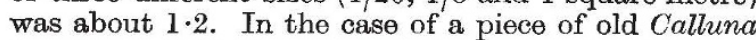
heath in which there were only six species of flowering plant, 76 per cent of the cover being provided by Calluna, while Empetrum nigrum and Vaccinium myrtillus formed the remainder, the calculated number of individuals expected from Williams' formula was $3,000^{\prime}$ per square metre. The actual number was not recorded, but the calculated number is obviously absurd.

Thus the index of diversity is apparently not applicable to numbers of plant species present in quadrats. The reason would seem to be that the formula takes no account of the amount of space occupied by the individual. In the insect populations first studied by Williams, sample size was defined by number of individuals-a fundamentally different method of sampling from that of taking quadrats, in which sample size is defined by area. The method of sampling an insect population comparable to taking a quadrat would be to take all the insects present in a given volume of space at any instant. While Williams' formula and the index of diversity derived from it may be valuable in some cases, it appears that the theoretical basis of its application needs further study.

Imperial Forestry Institute,

E. W. JONES. Oxford.

${ }^{1}$ J. Ecol, 32,1 (1944).
I HAVE read with interest Mr. Jones's letter on the results of the application of the logarithmic series and the index of diversity to his quadrat observations. I completely agree with him that the theoretical basis requires further study; as also does the field application to both animals and plants. The object of my publication, which gave a few cases selected over a wide field, was to stimulate the production of new data whether in support of the theory or not.

The essential difference between sampling in insects based on numbers and in plants based on area was emphasized by me both in the paper quoted and in an article in Nature $^{1}$. The application to botanical problems of a theory based on numbers of individuals was justified on the assumption that within one ecological formation the number of individuals could usually be considered as proportional to the area. This is only true if the size of the area sampled is large enough to contain a fair sample of the vegetation. In spite of this difficulty, I was surprised to find that in the sets of data that I collected almost at random from botanical literature, the results were consistent with the logarithmic series, and gave not unreasonable estimates of the numbers of individuals on a quadrat. If Mr. Jones's figures do not so fit, then it will be interesting to find the reason.

Even in animals, however, it is not always necessary to know the exact number of individuals in order to apply many of the general principles of the log series, including the index of diversity. For example, if two random samples of insects from the same population by the same method give $x$ individuals of 40 species and $3 x$ individuals of 60 species, then it is possible to say that $\alpha=\frac{60-40}{\log _{\diamond} 3}=18 \cdot 2$, without knowing the value of $x$, except that it must not be too small. This very closely resembles the case of two quadrats of size 1 and 3 units with an unknown number $x$ of individuals on each unit area.

In the limits of a letter, Mr. Jones cannot give all the essential data, but I will take first his statement about quadrats of $1 / 20,1 / 8$ and 1 square metre giving $\alpha=1 \cdot 2$. This would suggest, on the basis of the $\log ^{*}$ series, that if there were $x$ species on $1 / 8$ sq. $\mathrm{m}$. there should be approximately $x-1 \cdot 1$ on $1 / 20 \mathrm{sq} . \mathrm{m}$. and $x+2 \cdot 5$ on 1 sq. m. $\left(x-\alpha \log _{e} 2 \cdot 5\right.$, and $x+$ $\left.\alpha \log _{a} 8\right)$. On the other hand, his data for several quadrats of $1 / 8 \mathrm{sq}$. m. on the same area giving $\alpha=7$ would imply that if there were $x$ species on $1 / 8$ metre, there would be about $x+14.5$ on $8 \times 1 / 8$ sq. metres; in other words, that eight separated quadrats of $1 / 8$ sq. metre gave 12 species more than 1 sq. metre in a block. I suggest that the obvious explanation is that there was a strong aggregation of plants of each species in groups. When this is occurring, there will be very great differences between single small quadrats both in number of species and number of individuals, and one would not expect conformity with the log series unless the size of the sample was considerably increased. It is pointed out in the paper quoted that the application of the $\log$ series to quadrats is only justified if the number of individuals in the quadrat is large compared with $\alpha$. In the locality studied by Mr. Jones it seems likely that an area of 1/20 m., with plants as large as Calluna, might easily contain only one or two individuals.

Whether or not the whole conception of the log series could be extended to allow for aggregation, that is, to include a measure of it, I do not know, 